\title{
Les prénoms de l'an II et les autres :typologie des attributions de prénoms dans la France en révolution
}

\section{Raphaël Bange}

\section{(2) OpenEdition}

Journals

Édition électronique

URL : https://journals.openedition.org/ahrf/127

DOI : 10.4000/ahrf.127

ISSN : 1952-403X

Éditeur :

Armand Colin, Société des études robespierristes

Édition imprimée

Date de publication : 1 décembre 2000

Pagination : 61-86

ISSN : 0003-4436

\section{Référence électronique}

Raphaël Bange, «Les prénoms de l'an II et les autres :typologie des attributions de prénoms dans la France en révolution », Annales historiques de la Révolution française [En ligne], 322 | octobre-décembre 2000, mis en ligne le 21 février 2006, consulté le 23 avril 2022. URL : http://journals.openedition.org/ ahrf/127 ; DOI : https://doi.org/10.4000/ahrf.127

Ce document a été généré automatiquement le 23 avril 2022.

Tous droits réservés 


\title{
Les prénoms de l'an II et les autres :typologie des attributions de prénoms dans la France en révolution
}

\author{
Raphaël Bange
}

1 Les études prenant pour cadre la Révolution française représentent une part très importante dans la liste des travaux consacrés à l'histoire des prénoms. Le "phénomène" révolutionnaire a en effet déjà été étudié en long et en large, et même parfois en travers... Sujet rebattu, et pourtant toujours d'actualité le débat sur la méthode sans cesse renouvelé, le doute sur la signification des chiffres calculés par certains auteurs, l'absence d'analyse globale pleinement convaincante sont autant de raisons de retourner y voir de plus près autant de stimulations aussi, car les critiques et remises en question formulées ces dernières années par différents auteurs débouchent en même temps sur des perspectives nouvelles... Les prénoms révolutionnaires n'ont pas encore dit leur dernier mot!

2 On nous pardonnera donc d'avoir décidé de privilégier ici "l'événementiel”, de nous en tenir principalement à la face émergée de l'iceberg, au rebours de certaines tendances parmi les plus novatrices apparues récemment (et illustrées par d'autres auteurs dans ce même numéro). Mais centrer l'étude sur "les prénoms de l'an II" ce n'est pas pour autant renoncer à étudier aussi les " autres prénoms" attribués aux enfants, ceux dont le choix ne témoigne apparemment d'aucun changement par rapport aux pratiques des époques précédentes la quête d'une définition adéquate des prénoms révolutionnaires impose en effet un effort de classification qui ne peut qu'englober la totalité du corpus des prénoms en usage pendant la Révolution, les anciens comme les nouveaux, les plus anodins comme les plus explicites.

3 L'une des principales difficultés étant que chacun a tendance à voir midi à sa porte, il était indispensable de dépasser le strict cadre monographique. Non que nous ne soyons persuadés des vertus de la monographie, bien au contraire, mais ce type 
d'approche ne prend réellement toute sa valeur que lorsque elle se fonde sur une méthode appropriée et une connaissance suffisante du contexte général... La présente étude s'appuie essentiellement sur les recherches que nous avons menées dans le cadre de notre Doctorat, c'est-à-dire le dépouillement des registres de baptême et de naissance d'une cinquantaine de villes françaises comptant plus de 10000 habitants en l'an II, sachant que notre effort a principalement porté sur les villes les plus peuplées à l'époque ${ }^{1}$. Afin de ne pas nous focaliser sur les seuls milieux urbains, nous utiliserons également les données recueillies lors de notre travail de maitrise sur les 133 communes du district du Beaujolais ${ }^{2}$, et nous puiserons aussi quelques points de comparaison dans les nombreux travaux publiés sur d'autres localités et d'autres régions. Cependant, cette contribution se veut avant tout méthodologique : notre but ne sera pas de dresser un bilan des appellations révolutionnaires à travers la France mais de proposer une typologie des différentes manifestations de ce phénomène, typologie qui pourra ultérieurement servir à une synthèse globale sur cette question des prénoms de la Révolution.

4 Nous commencerons cet exposé par une première série de graphiques retraçant mois par mois l'évolution de la fréquence des prénoms révolutionnaires dans l'état civil de onze grandes villes entre 1793 et l'anIII (planche1, voir page suivante). Pour la composition de ces courbes, quels sont les prénoms qui ont été retenus comme révolutionnaires et ceux qui ne l'ont pas été On sait que le sujet est vaste et soulève de nombreux problèmes de méthode. Afin d'expliciter brièvement les choix que nous avons été amené à faire, précisons les deux critères fondamentaux qui ont été retenus : d'une part un critère idéologique, d'autre part un critère d'usage.

5 Critère idéologique : le problème de savoir si tel ou tel prénom possède un rapport avec les valeurs et les idéaux révolutionnaires est une question qui a déjà été souvent formulée. Sans doute les historiens doivent-ils arrêter de vouloir y répondre en faisant appel à leur propre subjectivité, pour retourner plutôt aux documents de l'époque et tenter de saisir ces valeurs fortes à travers les traces laissées par différentes manifestations contemporaines de l'attribution des prénoms révolutionnaires :

-la liste de tous les noms qui sont attestés comme toponymes révolutionnaires ${ }^{3}$;

-la liste de ceux qui figurent dans la nomenclature du calendrier républicain ${ }^{4}$;

8 -le programme des fêtes officielles célébrées par la République ;

9 -la liste des noms - héros ou valeurs morales - qui sont inventoriés dans les almanachs républicains publiés pendant cette période ${ }^{5}$;

10 -la liste des héros militaires dont le nom est gravé sur la colonne du Panthéon ;

11 -la liste des personnages historiques cités dans les discours prononcés à l'Assemblée ${ }^{6}$; etc ${ }^{7}$. 


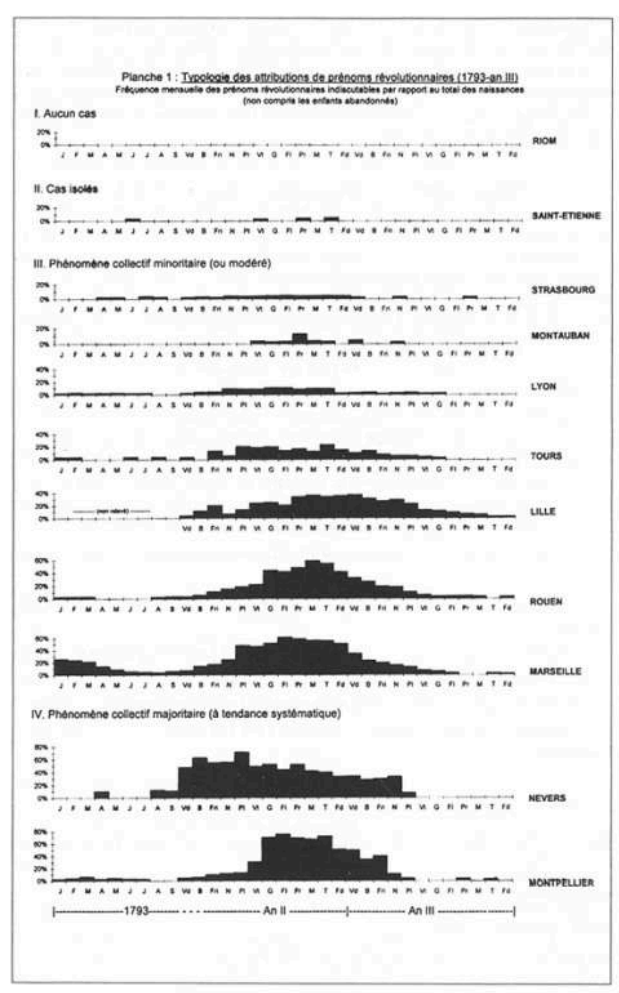

$12 \mathrm{Au}$ bout du compte, les valeurs qui sont le plus souvent revendiquées dans ces documents peuvent donc être caractérisées comme chargées d'une forte détermination révolutionnaire, alors que le lien idéologique sera par contre relativement faible pour celles qui n'apparaissent qu'une seule fois.

Quant au critère d'usage, cela signifie que pour chaque référence ou type de référence, nous nous sommes posé la question de savoir s'il s'agissait de prénoms ayant déjà été utilisés ou non dans les actes de baptême d'avant 1789, et si oui, quelle était leur diffusion.

Le postulat de départ est que n'importe quel nom de saint figurant au martyrologe peut être susceptible d'avoir été un jour utilisé comme nom de baptême, a fortiori s'il s'agit d'un saint vénéré quelque part en France. Et effectivement, si on prend par exemple le cas de sainte Pome, vierge à Châlons-sur-Marne, fêtée le 27 juin, Pome se retrouve donné quelquefois comme nom de baptême dans les registres paroissiaux de châlons, couramment aussi orthographié Pomme. On peut donc considérer qu'il s'agit d'un prénom tout à fait traditionnel en Champagne. Jusqu'à preuve du contraire, cela reste cependant un prénom d'usage strictement local ou régional, et si on vient à trouver le prénom Pomme à Marseille ou à Toulouse en 1794, la détermination ne sera pas automatiquement la même qu'à Châlons. Il faut alors s'interroger s'il n'y aurait pas de mère de famille marseillaise ou toulousaine déjà porteuse de ce prénom, ou bien si ce choix ne serait pas à mettre en relation avec la date de la fête de la sainte, le 27 juin. En l'occurrence, à Marseille et Toulouse, les sources dont nous disposons ne mentionnent pas l'existence de ce prénom dans les actes de baptême d'avant la Révolution et comme il n'apparaît que dans les premiers jours de brumaire - ce qui correspond à l'emplacement de la Pomme dans le calendrier républicain - ce choix devra donc être considéré comme révolutionnaire à part entière. 

Régime dans plusieurs régions différentes, par exemple Olive à Paris, à Nantes, à Marseille, etc., sa détermination initiale sera en revanche systématiquement considérée comme traditionnelle dans l'ensemble du pays, et ce, même si ici ou là il se trouverait des localités où ce prénom ne sera encore jamais arrivé. Dans certains cas, une détermination révolutionnaire pourra cependant venir se surajouter à la détermination traditionnelle initiale, par exemple si Olive venait à être utilisé en l'anII en relation chronologique directe avec l'application du calendrier républicain. il est possible de faire la part des choses et classer les unités prénominales suivant qu'elles renvoient exclusivement à la Révolution ou à la tradition, ou bien aux deux à la fois, voire à aucune des deux. En cas de surdétermination ou d'indétermination, il reste la possibilité de se reporter au contexte précis dans lequel le prénom est choisi et de proposer une interprétation au cas par cas, comme nous venons de le montrer pour Pomme $^{8}$. Si l'ambiguïté subsiste, le mieux est encore de renoncer à vouloir faire entrer un prénom de force dans un camp ou dans un autre. Nous verrons que le maintien de certaines catégories intermédiaires dans la classification des prénoms (références littéraires, noms susceptibles d'équivoque, prénoms “en progression"...) ne représente pas un handicap, loin s'en faut, à une bonne compréhension des choix de prénoms effectués pendant la Révolution.

Pour établir cette première série de graphiques, nous n'avons cependant retenu que les prénoms comportant au moins une référence révolutionnaire indiscutable, en laissant provisoirement de côté tous ceux qui étaient un tant soit peu déjà connus sous l'Ancien Régime, attestés comme noms de baptême dans différentes régions de France: cela concerne non seulement des exemples classiques comme Rose ou Victoire, mais aussi des cas comme Lucrèce, Scipion ou Hercule. En quelque sorte, ces graphiques ne donnent donc que le taux minimum de prénoms révolutionnaires. Mais il est indispensable de démarrer sur des bases aussi rigoureuses au départ pour pouvoir raisonner sur des valeurs sûres, quitte à élargir le propos par la suite en analysant le parcours d'autres types de prénoms.

18 Dernières précisions: nous n'avons pas distingué les sexes dans le calcul des pourcentages qui ont servi à l'élaboration de ces premiers graphiques. Quant à la catégorie particulière des enfants exposés ou abandonnés, dont la prénomination incombe habituellement à l'administration et présente donc un caractère quelque peu "officiel", elle a été laissée de côté. Pour une étude de l'acculturation des populations, il est évidemment plus instructif de s'intéresser en priorité aux prénoms choisis par les familles plutôt qu'à ceux qui ont été directement imposés par l'officier public.

19 La typologie que nous proposons est fondée essentiellement sur la répartition et la plus ou moins grande fréquence des prénoms révolutionnaires dans les actes d'état civil de la Révolution. Au vu des graphiques (planche1), il est aisé de distinguer plusieurs types de cas

20 -D'abord les communes où l'on ne relève pas le moindre prénom révolutionnaire indiscutable c'est le cas notamment à Riom, chef-lieu de district du Puy-de-Dôme, le premier graphique de la série il est vide puisque aucun prénom révolutionnaire sûr n'a été enregistré tout au long de la période considérée. 
21 Ce genre de cas n'est pas forcément le plus évident à interpréter l'absence totale de prénoms révolutionnaires signifie-t-elle une attitude caractérisée de rejet des valeurs républicaines Est-ce plutôt une preuve d'ignorance $\mathrm{Ou}$ bien simplement de l'indifférence vis-à-vis d'une pratique particulière, que certains militants ont pu juger inutile voire inopportune Difficile de trancher, même si, dans le cas précis présenté ici Riom étant la patrie de Gilbert Romme, l'un des instigateurs du calendrier républicain -, il est particulièrement intéressant de constater que l'on n'y enregistre aucun prénom révolutionnaire entre 1793 et l'anIII, alors même qu'il y a eu environ un millier de naissances déclarées dans cette ville au cours de cette période (sans compter les enfants exposés).

22 -Passons au second type de cas, illustré par la ville de Saint-Étienne c'est lorsque les prénoms révolutionnaires existent mais n'apparaissent que de manière isolée, comme une initiative réservée à quelque personnalité locale. Ici par exemple, le premier cas enregistré, en juin 1793, concerne la fille du maire, et certains prénoms révolutionnaires de l'anII mettront en scène d'autres notabilités politiques locales ${ }^{9}$. Au total, les prénoms significatifs se comptent sur les doigts d'une seule main. Ils n'ont donc rencontré pratiquement aucun écho auprès de la population. Pour une ville de cette importance, il semble que l'on puisse conclure à un réel échec.

23 -Et puis viennent les communes où l'état civil comprend un certain nombre de cas de prénoms révolutionnaires, suivant une répartition plus ou moins régulière mais qui témoigne au moins d'une certaine continuité dans le temps on peut alors parler de phénomène collectif, et tenter de mesurer l'ampleur du phénomène en question.

24 Nous avons donné sur cette figure un certain nombre d'exemples de villes où s'observent de tels phénomènes. Les villes représentées ont été classées suivant l'importance qu'y obtiennent les prénoms révolutionnaires, depuis le cas de Strasbourg où ceux-ci ne culminent jamais qu'à $3 \%$ des naissances, jusqu'à Nevers ou Montpellier où leur fréquence atteint certains mois 60 à $70 \%$ (et encore, en se limitant aux seuls cas indiscutables). Comme on peut le constater, nous avons distingué deux grands types de communes, d'un côté (type 3) celles où l'attribution de prénoms révolutionnaires reste un phénomène sinon toujours minoritaire du moins relativement modéré (même au plus fort de la vague, ces prénoms-là ne dépassent que rarement la barre de $50 \%$ des naissances), et de l'autre (type 4) les communes où le mouvement des prénoms révolutionnaires présente beaucoup plus d'ampleur, au point de devenir largement majoritaire à certaines périodes, quand il ne prend pas tout simplement une tournure systématique.

L'existence de ce dernier cas de figure amènera nécessairement à reposer la question de la spontanéité des choix de prénoms. D'ores et déjà, on voit que la ligne de séparation entre les types 3 et 4 ne passe pas uniquement par une différence de pourcentages, et il conviendra de renoncer à une approche purement quantitative pour faire intervenir d'autres critères d'analyse susceptibles d'expliquer de quoi il en retourne. La comparaison de ces différents profils offre d'ailleurs un aperçu fort suggestif : une opposition très nette se dessine entre deux types de communes, d'une part celles qui offrent une courbe plutôt régulière - on perçoit le phénomène en train de se développer, arriver à maturation puis retomber progressivement - et d'autre part les villes qui présentent un profil plus accidenté, avec des écarts parfois considérables d'un mois à l'autre: d'un côté la régularité de Rouen et de Marseille, de l'autre les sautes d'humeur de Nevers ou de Montpellier. 

mouvement des prénoms révolutionnaires. Il n'est pas de notre propos d'entrer ici dans les détails, aussi nous nous bornerons à résumer les principales tendances qui ressortent de la comparaison de ces différentes villes. partout à l'automne 1793, c'est-à-dire dans les premiers mois de l'an II. Certes, dans la quasi-totalité des villes étudiées, les nouveaux prénoms ont fait leur apparition dès le début de l'année 1793, ou parfois même avant. Mais les précurseurs de cette période n'ont guère d'imitateurs et la fréquence des choix révolutionnaires ne décolle pas vraiment. Seule Marseille fait figure d'exception (près de $25 \%$ de prénoms révolutionnaires dans les actes de janvier 1793), il est vrai qu'ici la tradition des Liberté et Brutus remonte aux Fédérations de $1790^{10}$. Mais à Marseille comme ailleurs, la crise fédéraliste du printemps à l'été 1793 a été marquée par un net reflux des appellations révolutionnaires. Il faut donc attendre l'automne - et le nouveau calendrier - pour que le déclenchement se produise enfin, les mois de brumaire ("B" sur le graphique) et frimaire ("Fri") se révélant comme une période décisive dans la grande majorité des villes étudiées (22octobre - 20décembre 1793). Chronologiquement, la diffusion des prénoms révolutionnaires dans les actes de naissance s'inscrit donc tout à fait en parallèle au "courant déchristianisateur" qui déferle alors aux quatre coins du pays. Dans ce contexte, une variation de dates de quelques semaines prend un relief particulier, et l'on remarquera que les habitants de Nevers se distinguent en adoptant massivement les prénoms révolutionnaires dès le premier mois du calendrier républicain ("Vd" pour vendémiaire), avec une avance certaine sur le reste du pays (et même sur la capitale $)^{11}$. Fouché y avait prêché l'exemple lors du baptême civique de sa fille sur l'autel de la patrie le 10août $1793^{12}$.

La chronologie des attributions de prénoms révolutionnaires obéit cependant à sa propre logique et ne se limite pas à la courte période du culte de la Raison et des mascarades antireligieuses, mais continue au contraire de prendre de l'ampleur au fil des mois. De manière remarquable, les maxima urbains se situent presque toujours au printemps et à l'été 1794 c'est-à-dire entre germinal ("G") et thermidor (“T") de l'an II, accompagnant (ou devançant) les célébrations à l'Être Suprême. Le tournant politique de thermidor ne provoque pas une nette cassure dans l'attribution de ces prénoms. Certes les courbes s'inversent inexorablement, mais dans certaines villes le reflux avait déjà commencé avant cette date.

Généralement, on observe encore un fort prolongement sur les premiers mois de l'anIII (dans une ville comme Lille c'est d'ailleurs à ce moment-là seulement que les meilleurs scores sont atteints), parfois jusqu'à l'hiver voire le printemps 1795. C'est un point à souligner, car on voit bien que si on limite le dépouillement des actes en s'arrêtant au dernier jour de l'anII, non seulement on laisse échapper un bon quart des prénoms révolutionnaires mais encore on perd une bonne part de compréhension du phénomène. Le constat serait encore plus parlant à l'échelle d'un district rural, ainsi celui de Villefranche-en-Beaujolais : sur vingt-six communes ayant connu au moins un prénom révolutionnaire, il y en a douze, soit presque la moitié, qui n'enregistrent aucun cas en l'anII dans trois cas, le ou les prénoms révolutionnaires datent du début 1793, dans cinq autres ils n'apparaissent qu'en l'anIII et même plutôt à la fin de l'anIII, et il reste encore quatre communes retardataires qui ne connaîtront leur premier cas significatif qu'en l'anIV ou même en l'anVI. Ce serait assurément faire une erreur de 
perspective que de se fonder uniquement sur ce qui se passe en l'anII, comme cela a malheureusement été le cas dans certains travaux récents. Évidemment, ce n'est plus Marat ou Liberté qui seront invoqués à ces dates tardives, mais rencontrer au village des choix comme Brutus, Vulcain, Décadine ou Bonaparte reste tout de même loin d'être anodin. Les choix révolutionnaires n'ont donc pas disparu du jour au lendemain, et on continuera d'en trouver sporadiquement au cours des saisons et années suivantes, non sans quelques "zéros" significatifs aux périodes de terreur blanche dans certaines grandes villes (ainsi à Marseille en prairial et messidor an III).

Pour asseoir notre démonstration sur des résultats fiables nous n'avons pour l'instant retenu que les seuls prénoms indiscutablement révolutionnaires. Mais les choix de prénoms sous la Révolution ne se limitent pas loin s'en faut à une alternative trop simple entre nom révolutionnaire ou nom traditionnel. Même dans le cas d'une ville où la population se montre largement réfractaire aux références républicaines, cela n'entraîne pas pour autant que tous les habitants de cette ville se contentent de redonner à leurs enfants les prénoms chrétiens déjà utilisés dans leur famille. Prenons l'exemple de Strasbourg, où les prénoms révolutionnaires ne dépassent pas 2 à $3 \%$ des naissances tout au long de la période. Nous nous sommes interrogé afin de déterminer sur quels types de choix se portaient les parents, et pour ce faire nous avons relevé non seulement tous les prénoms des enfants nés durant un an et demi (mars 1793 - fin anII) mais également ceux du parent et du témoin de même sexe ${ }^{13}$. À partir de ces données, si l'on compare les prénoms des garçons avec les prénoms de leurs pères, et réciproquement les prénoms des filles avec les prénoms de leurs mères, on peut tirer deux constatations très intéressantes.

La première, qui était attendue, concerne la permanence des mêmes unités dominantes d'une génération à l'autre : chez les enfants comme les parents, les prénoms les plus attribués sont Marie, Salomé (prénom biblique caractéristique de la population protestante), Madeleine et Catherine au féminin Jean, Jacques, Georges, François au masculin. Cette hiérarchie ne subit aucun bouleversement à l'épreuve de la Révolution.

En revanche, et ceci était moins prévisible au regard du faible score des prénoms créés par la Révolution, il existe aussi plusieurs prénoms très rares pour ne pas dire inexistants à la génération des parents et qui deviennent au contraire relativement fréquents dans les actes de naissance de la Révolution. Sophie par exemple on comptait en tout et pour tout 7 mères de famille qui portaient ce prénom, il est en revanche attribué à 99filles entre 1793 et l'anII. C'est encore plus net pour le prénom Caroline, pour lequel on passe de seulement 2 cas à la génération des parents, contre 67 cas à celle des enfants Chez les garçons, le phénomène est moins caractérisé mais on trouve tout de même Auguste qui passe de 2 à 20. Le parcours de ce prénom peut s'interpréter de plusieurs manières différentes, mais celui de Sophie ou Caroline est déjà pleinement significatif ces prénoms qui progressent de manière considérable sans avoir pourtant aucun lien direct avec la Révolution sont tout simplement les prénoms à la mode de cette fin de xviIIe siècle. Une mode qu'on ne peut circonscrire à la période révolutionnaire puisque tous ces prénoms, Sophie, Caroline ou bien encore Auguste, Joséphine, Alexandre et d'autres, relayés par le succès de certaines œuvres historiques et/ou littéraires, avaient déjà commencé leur carrière dans les registres paroissiaux des dernières années de l'Ancien Régime.

Alors que certains auteurs ont précisément tendance à qualifier de "phénomène de mode" l'apparition et la diffusion des prénoms révolutionnaires en l'an II, l'étude du 
cas strasbourgeois montre ainsi qu'il ne faut pas mélanger les choses. En matière de prénomination, les modes existent et ce n'est pas la Révolution qui les invente, mais si on compare les 3prénoms Brutus ou les 2 Viala qui figurent dans l'état civil de Strasbourg avec les 99Sophie et les 67 Caroline, on voit clairement où se situe la mode. Pour choisir de donner à son enfant un prénom comme Brutus ou Sans-Culotte il faut en réalité bien autre chose qu'une atmosphère culturelle favorable à l'innovation il faut d'abord et avant tout une réelle conviction politique.

L'existence de cette catégorie de prénoms qu'avec Philippe Daumas ${ }^{14}$ nous pouvons appeler "prénoms en progression" ressort donc particulièrement en évidence lorsque l'on compare les prénoms donnés aux enfants et ceux portés par leur entourage, que ce soit les parents ou les témoins mentionnés dans l'acte de naissance. Or, c'est également en se fondant sur cette comparaison qu'Alain Croix a tenté d'élaborer un critère d'analyse des prénoms jouant sur plusieurs registres à la fois: selon lui, des choix comme Rose ou Véronique pourraient être caractérisés dans un sens ou dans un autre suivant qu'ils apparaissent ou non en rapport avec les prénoms de l'entourage. Ayant démontré que $80 \%$ des Rose nantaises de l'an II sont des choix novateurs, sans rapport visible avec la famille, il conclut en estimant que ces $80 \%$ sont directement redevables à l'influence du calendrier républicain ${ }^{15}$. Cette conclusion nous parait trop rapide car si l'on en juge par les résultats signalés pour Strasbourg, tout laisse penser que des prénoms banals manifesteraient à Nantes la même progression que Rose, et l'on obtiendrait sans doute aussi près de $95 \%$ d'innovation dans les attributions du prénom Caroline... Si nous appliquons la méthode proposée à l'ensemble des actes de naissance de l'anII à Cherbourg, la proportion de prénoms choisis sans référence à ceux des parents et témoins mentionnés dans l'acte de naissance s'élève à 19 sur 29 pour Rose, mais la part d'innovation est encore plus élevée pour certains autres prénoms comme Victor (10sur 15), Aimable (9 sur 12), Adélaïde (9 sur 11), Auguste (12 sur 14), etc. ${ }^{16} \mathrm{Il}$ convient donc de ne pas confondre les prénoms en progression, fortement favorisés par le contexte de l'anII (notamment par la disparition partielle et provisoire des baptêmes religieux) et dont Rose fait partie, avec les autres prénoms plus caractéristiques qui surgissent brutalement dans l'état civil de la Révolution et représentent une réelle rupture par rapport aux pratiques antérieures. On peut clore temporairement ce dossier par une remarque en forme d'interrogation: les 700 familles nantaises qui se portent massivement sur le choix du prénom Rose pendant la Révolution ont-elles eu réellement un comportement plus novateur que les quelques dizaines de familles qui ont découvert ce même prénom avec trente ou cinquante ans d'avance, au cours du xviliesiècle? Cette remarque vaut assurément pour un bon nombre de prénoms limites, dont la carrière a commencé bien avant la Révolution...

L'intérêt accordé aux diverses catégories de prénoms existant dans les actes de naissance de la Révolution, y compris les références chrétiennes les plus traditionnelles, ne doit pas apparaitre comme un simple complément à l'étude des prénoms révolutionnaires. C'est au contraire une approche indispensable, a fortiori si l'on souhaite interpréter correctement la réalité des choix de prénoms à cette époque. La deuxième série de graphiques, destinée à préciser la distinction que nous établissons entre phénomènes collectifs de type 3 et 4 ("phénomène collectif modéré" / "phénomène majoritaire à tendance systématique"), illustre l'importance d'une analyse globale étendue cette fois à tous les types de prénoms (planche 2, voir page suivante). 
Pour la précision du propos, nous avons désormais séparé les cas en fonction du sexe (colonne de gauche les filles, colonne de droite les garçons).

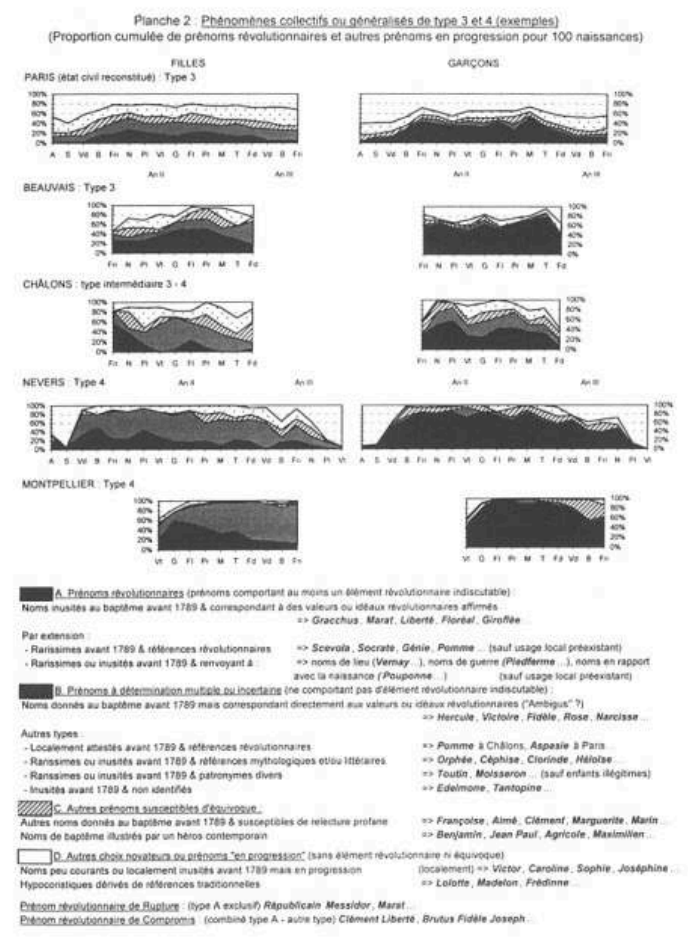

En plus de la catégorie de prénoms que nous cataloguons comme "révolutionnaires" indiscutables (catégorie "A", la même que celle figurant précédemment dans la première série de graphiques), nous avons fait intervenir trois autres nuances renvoyant à trois autres séries de prénoms caractéristiques. Nous avions retenu au départ bien davantage de catégories intermédiaires, mais au vu des résultats il a été possible de procéder au regroupement de prénoms présentant une évolution relativement similaire ( $c f$. la classification schématique que nous indiquons en-dessous des graphiques) :

-les prénoms qui répondent à une détermination multiple ou incertaine (catégorie “B"), comme Rose, Victoire ou Virginie pour nommer les plus fréquents, ou Pomme à Châlons pour citer un exemple de prénom dont la détermination habituelle est localement contrebalancée par un usage plus ancien. S'y ajoutent quelques références mythologiques ou purement littéraires, ainsi qu'une poignée de prénoms indéterminés ne renvoyant à rien de connu et n'ayant pu être identifiés.

-des prénoms dont la détermination habituelle est traditionnelle mais qui, dans un contexte particulier, peuvent se trouver remotivés dans un sens plus en rapport avec les idéaux révolutionnaires (catégorie "C") : quoique absent du calendrier républicain, le nom de Marguerite perd par exemple de sa banalité et retrouve un côté champêtre prononcé si on le retrouve tout à coup au milieu d'une série de Narcisse et de Pervenche. Les prénoms qui rappellent les noms de baptême de certaines personnalités fonctionnent sensiblement de la même manière : Benjamin ou Maximilien sont déjà des prénoms d'usage courant mais dans un certain contexte ils peuvent passer pour des choix délibérés en l'honneur de Franklin ou de Robespierre... (une attitude cependant beaucoup plus modérée que d'appeler son fils Franklin ou L'Incorruptible!). 

de Nevers et de Montpellier rendent typiquement compte d'un phénomène collectif généralisé ou systématique (type 4). On voit en effet très nettement que si l'on ajoute aux prénoms révolutionnaires les trois autres catégories de prénoms définies prénoms mixtes, prénoms équivoques et prénoms en progression - on arrive ici à couvrir la totalité des naissances. Que ce soit à Nevers ou à Montpellier, la visualisation graphique est très parlante, la courbe reste en effet collée pendant plusieurs mois au plafond des $100 \%$.

44 seraient d'apparence plutôt anodine, ainsi Romain Angélique ou Marin, mais qui, au vu du contexte local, reçoivent une connotation tout à fait différente de l'usage habituel. Marin par exemple devient à Nevers le prénom fétiche des mariniers sur la Loire, alors que c'était un prénom inusité dans ce milieu avant la Révolution. Il s'y ajoute une petite proportion de prénoms en progression - par exemple Caroline, Laure, Adèle - encore 
inconnus à la génération des parents, et enfin, pour compléter le tableau, on trouve aussi quelques diminutifs comme Madelon ou Toninette, lesquels n'ont strictement rien de créations révolutionnaires mais rejoignent pourtant, d'une certaine manière, le processus de rupture avec les pratiques de l'époque précédente : eux aussi sont en effet des formes inédites dans les actes officiels avant 1793 le curé chargé du baptême aurait systématiquement corrigé en Madeleine ou Antoinette... Au bout du calcul, on s'aperçoit qu'à Nevers comme à Montpellier les prénoms traditionnels du style Marie, Jean ou Jacques ont totalement disparu, et ce pendant plusieurs mois. On ne trouve plus un seul enfant prénommé de cette manière, comme si on avait affaire à une volonté délibérée de purger le répertoire des prénoms de tout ce qui rappellerait trop ostensiblement les pratiques traditionnelles de l'Ancien Régime, marquées du poids des références chrétiennes.

Voilà évidemment une situation typique du processus révolutionnaire : faire table rase du passé, abolir pour créer du neuf. En matière de prénomination, c'est une tendance que les chercheurs ont eu un peu tendance à oublier pour s'intéresser aux seuls aspects les plus caractéristiques de la création révolutionnaire. Ce rejet des pratiques de l'Ancien Régime est pourtant une tendance qui prend de multiples aspects, Jean-Pierre Lethuillier en a présenté un autre en mettant en évidence la diminution du nombre d'unités prénominales en l'anII dans les régions qu'il a étudiées ${ }^{19}$.

Il peut cependant arriver que le mouvement d'attribution des prénoms révolutionnaires prenne localement une tournure systématique sans pour autant entraîner la totale disparition des références traditionnelles. Les unités nouvelles ne se substituent pas toujours aux anciennes, elles leur sont parfois tout simplement associées, formant ce que l'on a coutume d'appeler un "prénom de compromis". C'est notamment ce qui se produit à Arras (non représentée sur ces graphiques), où la courbe reste quasiment à $100 \%$ pendant plusieurs mois (floréal an II - brumaire an III) si on cumule des prénoms comme Râteau Floréal ou Paul Brutus avec des choix beaucoup moins marqués comme Théophile, Benjamin Narcisse et Antoine Désiré... Ces derniers cas (comportant des prénoms équivoques ou en progression) ne sont pas, loin s'en faut, des prénoms révolutionnaires, mais ils reçoivent un sens bien particulier lorsque l'on s'aperçoit que pendant toute cette période, il n'y a plus aucun enfant banalement prénommé Pierre Henri ou Catherine Joseph, comme c'était encore couramment le cas quelques mois plus tôt. Les prénoms donnés dans les actes de naissance d'Arras en 1794 composent donc une variante d'un phénomène collectif généralisé de type 4 , où chaque enfant semble tenu de porter au moins un prénom faisant référence de près ou de loin à une valeur politique ou morale appréciée des républicains.

Conformisme? Phénomène d'imitation et d'engouement collectif? Ou bien plutôt pression de l'officier public ou d'autres responsables Sans doute y a-t-il un peu de chaque. Mais il ne faut pas se cacher les yeux: dans un cas où la prénomination des enfants prend un tour aussi systématique, cela ne peut être qu'artificiel. Dans les trois villes que nous avons citées, la marge de manœuvre des parents est visiblement réduite et la liberté de choix est sujette à caution.

Ce qu'il importe tout de suite de préciser, c'est que l'on n'y observe aucune irrégularité dans l'enregistrement des actes (à une seule exception près, mais qui fera parler d'elle ${ }^{20}$, pas de ratures ou de surcharges intempestives, très peu d'actes qui feront ultérieurement l'objet d'une demande de rectification en justice ${ }^{21}$, une répartition des témoins demeurant tout à fait normale (les gens continuent de déclarer leur enfant en 
présence de leurs voisins ou de leurs familles), et par ailleurs il peut y avoir plusieurs officiers d'état civil différents qui se succèdent au cours de la période sans que cela ne change quoi que ce soit au comportement des parents. Dans ces conditions, parler de contrainte organisée serait assurément spéculatif, même si on peut se demander quels ont pu être les moyens d'incitation mis en œuvre pour arriver à de telles séries de prénoms paraissant tous sortis d'un même moule. Tout au plus peut-on remarquer que la "pression" a tendance à baisser au fur et à mesure que les mois passent: sur les graphiques de Nevers et de Montpellier, si la proportion cumulée de nos quatre catégories de prénoms se maintient durablement à $100 \%$, les prénoms les plus engagés du début de période ont tout de même peu à peu cédé du terrain sur les choix les plus modérés. Comme une façon de sauver les apparences...

Une telle incitation a pu exister dans d'autres villes sans pour autant donner des résultats aussi tranchés. À Beauvais par exemple, le mouvement des prénoms révolutionnaires connaît là aussi une forte amplitude, accompagnée de nombreux choix équivoques ou en progression ( $c f$. graphique), mais à aucun moment les prénoms traditionnels ne disparaissent complètement des actes de naissance et on continue épisodiquement de rencontrer des Marie Madeleine ou des Jean Joseph. La liberté collective des parents quant aux choix du prénom de leur progéniture ne semble donc pas pouvoir être ici remise en doute, et le cas de Beauvais est à ranger parmi les phénomènes de type 3, par opposition aux systèmes généralisés de type 4 comme Nevers ou Montpellier. La ville de Marseille présenterait une situation analogue à celle de Beauvais, à une différence notable: si les Joseph et les Madeleine ne posent aucun problème aux officiers publics, les prénoms de Louis(e) et d'Antoinette semblent quant à eux avoir été systématiquement proscrits, on n'en rencontre plus un seul entre nivôse et fructidor anII

Les "séries" de prénoms se conformant à un même modèle local ne sont pas toujours enregistrées à l'échelle d'une commune entière. Le phénomène se concentre parfois à une catégorie particulière de la population (exemple caricatural: les enfants nés à l'hôpital et nommés par l'administration suivant un mode systématique) ou aux actes de naissance d'un quartier précis. Dans certaines villes, comme à Rochefort et à Moissac, on n'observe l'existence d'un phénomène généralisé que dans le registre de naissance des enfants nés intra-muros, alors que les prénoms révolutionnaires demeurent au contraire épisodiques et minoritaires pour les naissances du faubourg.

51 Bien évidemment, le conformisme a pu jouer aussi au micro-échelon des relations de travail ou de voisinage, mais il s'agit alors d'un phénomène d'une tout autre nature que les séries orchestrées avec le concours plus ou moins tacite des autorités. Certains cas intermédiaires restent cependant difficiles à interpréter, comme celui de Châlons (en Champagne) dont nous avons donné également la représentation graphique: en cumulant les différentes catégories de prénoms en vogue, le pic des $100 \%$ de naissances est atteint à plusieurs reprises entre l'hiver et l'été 1794, mais sans pour autant que la courbe se maintienne à ce niveau comme dans les autres graphiques caractéristiques du type 4. Cela traduit sans doute les incertitudes et les hésitations des militants républicains locaux.

Lorsque la fréquence des prénoms révolutionnaires commence à grimper, il importe donc de se livrer à une analyse attentive des registres de naissance, en ayant conscience que s'il a existé ici et là des mouvements "encouragés" d'une manière ou d'une autre par quelque autorité locale, cela ne se traduit pas forcément par des choix 
spectaculaires: le plus souvent, on a pu s'accommoder de prénoms passe-partout comme Hyacinthe ou Fidèle, voire de noms de baptême plus ou moins rares mais n'évoquant pas trop ostensiblement la religion (Caroline, Rosalie...). En définitive, les phénomènes de prénomination systématique de ce type paraissent moins motivés par l'affirmation de valeurs reconnues comme républicaines que par le rejet des pratiques traditionnelles : ils manifestent assurément une réaction contre (ou une subversion de) la quasi-unanimité qui se dirigeait jusque-là dans l'autre sens, en faveur des références chrétiennes, quand l'enregistrement des baptêmes était effectué sous le contrôle vigilant du clergé.

Cette réaction peut encore s'exprimer différemment, en s'attaquant à la forme plutôt qu'au fond ainsi lorsque certains républicains décident de rejeter les prénoms multiples comprenant deux, trois ou quatre unités - considérés comme aristocratiques - pour s'en tenir à un prénom simple (ce qui rejoint finalement les directives du Comité d'instruction publique au sujet des changements à opérer dans les appellations de communes, préconisant d'adopter "des noms courts et sonores"). Pour quelques villes de notre corpus, on remarque non seulement une diminution mais une presque disparition des prénoms multiples, en un mouvement systématique plus ou moins durable mais qui n'entraîne cependant pas toujours le développement d'une prénomination révolutionnaire de type 4. Ainsi à Caen, presque tous les enfants nés entre messidor anII et floréal anIII ne reçoivent qu'un prénom, mais ils ne sont que cinq (sur un millier de naissances!) pour lesquels il s'agit d'une référence révolutionnaire indiscutable. L'état civil de Versailles présente une situation encore plus intéressante, quoique exceptionnelle : la règle de n'attribuer qu'un seul prénom aux nouveau-nés est ici explicitée dès l'enregistrement des premiers actes civils (le 28octobre 1792) ${ }^{22}$, et perdurera jusque vers la fin de l'anIV! La prénomination unique prend alors une tournure quasi officielle, résultant peut-être, à l'origine, d'une mauvaise compréhension de la loi par les officiers publics ${ }^{23}$. La contrainte qui s'exerce sur les parents au moment des déclarations de naissance n'entraîne pas pour autant une généralisation des prénoms révolutionnaires en l'anII (les graphiques versaillais se calqueraient plutôt sur le modèle parisien de type 3 , à un niveau légèrement inférieur). En revanche, les quelques prénoms doubles qui parviennent à filtrer de cet ensemble "uniciste" apportent de précieuses informations sur la notion de prénom composé à l'époque de la Révolution ${ }^{24}$.

54 Afin de pouvoir mener à bien une étude des prénoms pendant la Révolution, et faire la distinction entre les différents cas de figure qui se présentent, il est donc nécessaire de rester attentif à l'ensemble des prénoms attribués et à l'évolution séparée de chaque catégorie. La typologie d'ensemble que nous avons ainsi pu établir permet d'affiner la compréhension du phénomène des prénoms révolutionnaires dans sa diversité, plutôt que de s'en tenir aux sacro-saintes moyennes plaquées sur l'anII qui n'ont d'autre résultat que de brouiller la perspective. Plusieurs auteurs ayant étudié des villes présentant des situations de type 4 (par exemple Arras ou Nevers) semblent ainsi ne s'être pas même rendus compte du tour systématique pris par la prénomination locale et se sont contentés de donner des pourcentages bruts qui traduisent bien mal la réalité. L'historiographie des prénoms de la Révolution a malheureusement souffert de ce manque de clarté, comme de l'imprécision qui caractérise deux contributions méthodologiques majeures des années 1980, celles de Serge Bianchi et de Gérard Cholvy, qui ont eux aussi travaillé sur des phénomènes de type systématique ${ }^{25}$. 
Si la comparaison des différents profils nous a conduit à isoler des situations extrêmes où l'on peut nourrir des doutes sur la spontanéité des choix de prénoms, il convient aussi de ne pas en exagérer outre mesure l'importance. Le tableau ci-dessous indique comment les villes de plus de 10000 habitants se répartissent entre les quatre principaux types que nous avons dégagés. Pour ne pas s'intéresser uniquement aux villes, la seconde partie du tableau offre une comparaison avec la situation que nous avons pu observer en étudiant l'ensemble d'un district rural, celui de Villefranche-enBeaujolais.

Tableau 1 : Distribution de deux échantillons de communes suivant l'importance des attributions de prénoms révolutionnaires

\begin{tabular}{|l|l|l|l|}
\hline & Villes de $10000 \mathrm{~h}$ et + & Communes du district du Beaujolais \\
\hline \hline $\begin{array}{l}\text { Type } 1 \\
\text { (aucun cas) }\end{array}$ & $1 \quad(1,5 \%)$ & $107 \quad(80 \%)$ \\
\hline \hline $\begin{array}{l}\text { Type } 2 \\
\text { (cas isolés) }\end{array}$ & $1 \quad(1,5 \%)$ & $17 \quad(13 \%)$ \\
\hline \hline $\begin{array}{l}\text { Type } 3 \\
\text { (phénomène collectif modéré) }\end{array}$ & $57 \quad(82,5 \%)$ & $7 \quad(5,5 \%)$ \\
\hline \hline $\begin{array}{l}\text { Type } 4 \\
\text { (phénomène collectif généralisé) }\end{array}$ & $10 \quad(14,5 \%)$ & $2 \quad(1,5 \%)$ \\
\hline \hline Total de communes & $69 \quad(100 \%)$ & $133 \quad(100 \%)$ \\
\hline
\end{tabular}

Pour les villes de plus de 10000 habitants sur lesquelles nous disposons d'informations précises, c'est à l'évidence le type 3 qui est de loin le plus répandu. Les types 1 (Riom) et 2 (Saint-Étienne), caractérisés par l'absence totale ou quasi totale de prénoms révolutionnaires, sont rarissimes en milieu urbain, le type 4 concernerait à peu près $15 \%$ des villes, alors que le type 3 en regroupe plus de 80 . Ces statistiques portent sur 69grandes villes, c'est-à-dire qu'en plus de nos propres recherches nous nous sommes appuyé sur la consultation des monographies qui ont été publiées sur différentes communes. Il faut cependant avoir conscience que les chercheurs ont tendance à s'intéresser de manière privilégiée aux phénomènes les plus marqués (nous-mêmes avons accordé une attention particulière aux communes donnant lieu à des mouvements d'attribution systématique de prénoms révolutionnaires), et les types 1 et 2 paraissent un peu sous-évalués dans cette statistique urbaine, on en trouverait sans doute d'autres exemples si on se livrait à des recherches exhaustives sur l'ensemble des villes française de cette importance.

57 L'existence d'un mouvement collectif minoritaire / modéré est le cas le plus typique dans les villes, c'est aussi celui qui a l'avantage de poser le moins de problèmes d'interprétation. Lorsque dans une commune le choix d'un prénom a été conçu par une partie des habitants comme un moyen d'expression politique, il devient en effet intéressant de mesurer quelle est la proportion de parents qui acceptent de franchir le pas et manifestent de cette manière leur engagement pour les valeurs républicaines. 
Cela peut bien sûr recouvrir localement certains clivages politiques ou des différences de motivation individuelle (il y aura toujours des révolutionnaires convaincus qui jugeront inutile voire inopportun de choisir de tels prénoms, et réciproquement des opportunistes qui y trouveront un moyen commode de se donner bonne conscience, ou de paraître plus engagés qu'ils ne le sont). Mais au final, le phénomène dépasse ici de beaucoup le cas de conscience d'individualités isolées et en vient à concerner une partie plus large de la population. Les statistiques et les graphiques qu'on peut alors tirer des actes d'état civil permettent indubitablement de distinguer des villes globalement réfractaires (comme le sont Strasbourg ou Lyon) et d'autres au contraire plus réceptives (Paris, Lille, Rouen, Marseille...), mais à des degrés divers. La hiérarchie qui se dégage ainsi est très riche d'enseignements sur l'inégale acculturation politique de ces populations urbaines en Révolution.

Dix villes étudiées présentent un phénomène collectif généralisé de type 4, c'est-à-dire que les partisans des prénoms révolutionnaires y obtiennent une position majoritaire, laquelle se concrétise pendant plusieurs mois par des séries d'actes de naissance systématiquement orientés vers le choix de références républicaines, ou du moins de prénoms en rupture avec les habitudes locales. Ce cas de figure concernerait donc en l'an II une proportion non négligeable de grandes villes (environ 15\%), encore avonsnous incorporé dans ce total des cas intermédiaires comme Châlons et Saint-Quentin, où le taux de prénoms novateurs ou équivoques n'atteint que très ponctuellement le chiffre de $100 \%$. Remarquons que ces dix villes ne figurent pas parmi les plus peuplées du pays : seule Montpellier dépasse 30000 habitants à cette époque, Arras et Rochefort en ont plus de 20000 mais les autres communes concernées s'inscrivent plutôt entre 10000 et 12000 (outre Châlons et Saint-Quentin, il s'agit de Nevers, Niort, Moissac, Agen et Beaune $)^{26}$. Les villes de moindre importance sembleraient donc les plus touchées par ce phénomène, et nous pourrions déjà ajouter à cette liste plusieurs villes de moins de 10000 habitants comme Béthune et Corbeil, mais aussi Agde ${ }^{27}$, Largentière ${ }^{28}$, Brive ${ }^{29}$, Saint-Antonin-Nobleval ${ }^{30}$ ou encore Villefranche, la capitale du Beaujolais. Mais ce genre de cas devient en revanche nettement plus rare quand on s'intéresse uniquement aux communes rurales...

59 La comparaison à laquelle invitent les chiffres donnés pour l'ensemble du district du Beaujolais montre en effet une répartition fort différente de celle observée pour les villes. La grande majorité des communes du district se rangent ici dans le premier type de cas, c'est-à-dire que dans 107 communes sur 133 il n'y a eu aucun prénom révolutionnaire à noter, la population est passée complètement à côté du phénomène. Probablement y aurait-il une distinction supplémentaire à faire en s'intéressant cette fois-ci à la catégorie que nous avons laissée de côté dans cette étude, c'est-à-dire les enfants déclarés par l'administration. Dans certains chefs-lieux de canton par exemple, on arriverait à trouver un ou deux cas d'enfants abandonnés qui ont été nommés en référence aux modèles révolutionnaires, ce qui signale donc que ces modèles n'étaient pas totalement ignorés, du moins par les responsables politiques locaux. Mais aucun d'entre eux ne se signalera en donnant un prénom révolutionnaire à son propre enfant...

Quant au reste des communes beaujolaises, on en compte seulement 26 (soit 20\%) qui enregistrent au moins un prénom révolutionnaire indiscutable, encore s'agit-il généralement d'un seul cas isolé. Il serait naïf de s'étonner de la rareté des prénoms révolutionnaires au village, non seulement car l'environnement culturel n'y est 
évidemment pas le même qu'à la ville, mais déjà pour un critère purement statistique : dans certaines localités où l'on ne compte habituellement qu'une à deux naissances par mois, cela laisse relativement peu de chances qu'un déclic se produise! Nous ne sommes plus ici à la même échelle qu'une ville comme Saint-Étienne, et lorsqu'on trouve déjà un cas indiscutable dans un petit bourg ou un village, cela signifie assurément beaucoup en matière de diffusion des valeurs nouvelles. Il faut d'ailleurs avoir présent à l'esprit que l'attribution d'un prénom politique au fils du notable du coin représente déjà en soi un petit événement, qui sera certainement loin de passer inaperçu et laissera au contraire quelques traces durables dans la mémoire locale.

Dans le district du Beaujolais, la mise en évidence de prénoms révolutionnaires en série, c'est-à-dire de phénomènes collectifs locaux de type 3 ou 4 , se restreint ici à 9 communes sur 133, parmi lesquelles on retrouve surtout les plus peuplées du district, la capitale Villefranche mais aussi plusieurs importants chefs-lieux de canton comme Beaujeu, Tarare ou Belleville. Très peu de villages ont donc été réellement touchés par le phénomène. Hormis Villefranche, un seul autre cas où les prénoms de la Révolution tendent à s'imposer de manière systématique : le petit bourg d'Aigueperse, aux confins des Monts du Beaujolais, une commune où la fermeture de l'église avait suscité une petite "vendée" locale provoquant l'envoi d'une force armée par le comité de surveillance du district... ${ }^{31}$ Les études publiées par d'autres chercheurs confirment la rareté des "séries" de prénoms révolutionnaires enregistrées à la campagne, même si plusieurs cas ont été signalés en diverses régions, ainsi à Saint-Bonnet-de-Salers dans le Cantal $^{32}$, à Méasne (Creuse) et Lussac-les-Églises (Haute-Vienne) en Limousin ${ }^{33}$, à Gourgé près de Parthenay (Deux-Sèvres) ${ }^{34}$, à Quillebœuf et Écouis dans l'Eure ${ }^{35}$, ou encore à Mézilles dans l'Yonne ${ }^{36}$.

À notre sens, le critère le plus intéressant lorsqu'on étudie les campagnes consiste à considérer en bloc l'ensemble des communes d'un district, et à juger la pénétration des prénoms révolutionnaires en calculant la proportion de communes qui comptabilisent au moins un prénom révolutionnaire indiscutable. Pour le Beaujolais, nous avons vu que cela représente $20 \%$. L'intérêt serait évidemment de disposer de données similaires pour chaque région, et de fil en aiguille pouvoir dresser une carte de France suivant l'intensité de la diffusion des prénoms révolutionnaires en milieu rural. Le district de Villefranche paraît relativement peu concerné (par rapport aux campagnes de l'Essonne ou du Pas-de-Calais respectivement étudiées par Serge Bianchi et Albert Vion) ${ }^{37}$, mais nous manquons malheureusement de points de comparaison fiables avec d'autres régions. Pour le district de Compiègne, Jacques Bernet estime que la moitié des communes environ connaissaient au moins un prénom nouveau dans le département des Côtes-du-Nord, plus d'un tiers des communes rurales étudiées par Aline Trabut auraient vu l'attribution de prénoms révolutionnaires ${ }^{38}$. En élargissant les recherches, on trouvera sans doute aussi des régions qui se situeront à un niveau plus faible que le Beaujolais, comme cela semble le cas du département de la Côte-d'Or ${ }^{39}$. Il y a énormément à attendre de ce point de vue-là d'une étude étendue à l'ensemble des communes de France.

63 Une telle enquête permettra aussi de développer la cartographie des localités où l'attribution des prénoms révolutionnaires donne lieu au développement d'un phénomène collectif de type 3 ou 4 . Apparemment, on trouvera dans la plupart des départements quelques communes caractérisées par des séries systématiques comme celles de Nevers ou de Montpellier, plus souvent dans des villes que des villages. Même 
si cela ne devait représenter que $2 \%$ du total des communes, il sera particulièrement important de chiffrer, localiser et étudier avec précision les municipalités où un tel modèle a fonctionné. Non seulement pour pouvoir donner de ce phénomène une analyse plus complète que nous n'avons pu le faire ici, mais aussi à cause du poids que ces communes pèsent dans les statistiques lorsque l'on dresse l'inventaire des prénoms révolutionnaires pour le Beaujolais par exemple, la commune de Villefranche comptabilise à elle seule $60 \%$ des prénoms révolutionnaires attribués dans le district. Et si l'on s'en tient aux communes rurales, le cas hors norme d'Aigueperse regroupe à lui tout seul presque la moitié des prénoms indiscutables enregistrés dans les campagnes beaujolaises Ces résultats incitent au scepticisme quant à la représentativité d'études qui seraient menées sous forme de sondages, portant sur des localités déterminées au hasard: lorsque seulement 2 communes sur 133 présentent un caractère exceptionnel, il suffit que ces communes figurent ou non dans l'échantillon tiré au sort pour que le résultat final diverge du tout au tout...

Cette étude comparative, portant sur un échantillon de communes déjà important, aura permis tout à la fois de montrer la diversité des situations observées et en même temps de dégager certains caractères fondamentaux sur lesquels asseoir une typologie des attributions de prénoms à travers les différentes villes et régions de France. Car on ne peut comparer que ce qui est comparable les limites d'une approche purement quantitative imposent de recourir à d'autres critères, d'ordre qualitatif, qui prennent en considération les spécificités de la prénomination à l'échelon de chaque commune, d'autant que le poids du contexte local s'avère parfois déterminant dans la multiplication (ou au contraire l'absence totale) de prénoms en rapport avec la Révolution. En élargissant l'analyse à l'ensemble des prénoms figurant dans les actes de naissance de cette époque, on peut parvenir à mettre en évidence d'autres évolutions localement caractéristiques - progression de prénoms anodins ou équivoques, disparition de certains choix traditionnels-, d'apparence moins spectaculaire que la prolifération de prénoms directement issus de la Révolution, mais pourtant riches de signification pour une juste appréciation du phénomène.

Les différents cas de figure que nous avons présentés, regroupés en quatre types principaux, révèlent de forts contrastes dans le cheminement des prénoms révolutionnaires variations d'intensité d'un lieu à l'autre dans la fréquence de leurs apparitions à l'état civil, mais aussi différence de nature dans la réalité des choix effectués par les parents, l'adoption d'un prénom en rupture avec la tradition témoigne en effet d'un engagement bien différent suivant qu'il apparaisse localement isolé ou qu'il s'inscrive au sein d'un courant collectif plus ou moins développé, obéissant à un mouvement spontané ou au contraire "encadré" de quelque manière que ce soit... L'élaboration d'une typologie adaptée à rendre compte de cette disparité de situations est un préalable méthodologique indispensable à toute étude rigoureuse sur la question des choix de prénoms pendant la Révolution, a fortiori si l'on veut pouvoir en tirer des indications fiables au sujet de l'acculturation politique des populations. Le bilan partiel que nous avons esquissé ici apporte dans ce sens quelques premiers résultats concrets, qui contribuent à préciser et renouveler les connaissances sur la géopolitique de la France en Révolution. C'est dire tout l'intérêt que l'on peut attendre d'une enquête d'envergure qui viserait cette fois à couvrir la totalité du territoire national... 


\section{NOTES}

1.Pour le détail des villes étudiées, et davantage de précisions sur les résultats présentés dans cette communication, voir notre thèse de Doctorat (à soutenir prochainement) à l'Université de ParisI Panthéon-Sorbonne, sous la direction de Catherine Duprat. Notre corpus comprend notamment toutes les villes comptant plus de 25000 habitants vers 1794 (Atlas de la Révolution française, vol. 8 : Population, Bernard LEPETIT et Maroula SinARellis éds., Paris, 1995, tableau p.74), à l'exception de Nîmes, Metz, Rennes (voir les travaux dirigés par Jean-Pierre Lethuillier) et Nantes (bien étudiée dans l'enquête dirigée par Alain Croix : Brutus, Désir de la Paix et Jonquille. Les prénoms de la Révolution en Loire-Inférieure, Alain CRoIX et Pascale SCILBo éds., Nantes, 1993, 143 p.).

2.Raphaël BANGE, La déchristianisation dans le district de Villefranche-sur-Saône pendant la Révolution française : l'exemple des prénoms, mémoire de maîtrise sous la direction de Françoise Bayard, Université Lyon II, 1990, 236 p. Ce travail peut être consulté au Centre Pierre Léon, Université Lyon II, de même que d'autres maîtrises sur la question des prénoms révolutionnaires dans la région lyonnaise : Béatrice Cousin (ville de Lyon), Serge Maury (district de Lyon-Campagne), Marie-Claude Bonnefond (district de Roanne).

3.Roger de FiguèRE, Les noms révolutionnaires des communes de France. Liste par département et liste générale alphabétique, Éd. Sociétés de l'Histoire de la Révolution française, Paris, 1901. Quoique incomplet, cet index donne les changements de noms de plus de 3000 communes sur l'ensemble du pays et reste une référence irremplaçable.

4.James Guillaume, éd., Procès-Verbaux du Comité d'instruction publique de la Convention nationale, Paris, Imprimerie nationale, 1891-1906, reproduit le détail des différents projets de calendrier étudiés par le Comité d'Instruction publique ainsi que les variantes et corrections intervenues entre les différentes éditions de la version officielle.

5.D'autant que certains auteurs d'almanachs ambitionnaient d'influencer les choix de leurs contemporains, comme Jacquin et Dupin dans le préambule de leur Almanach du républicain : "Le coup le plus terrible et le plus décisif porté aux préjugés religieux, c'est le changement de calendrier grégorien [...] Les saints d'une république sont les citoyens qui l'ont bien servie. Brutus, Cassius, Caton, Aristide, voilà les patrons qui conviennent à des hommes libres." Nicoline Hörsch a donné une intéressante présentation de quelques almanachs politiques et d'autres sources de motivation possible des choix de prénoms révolutionnaires ("De Pierre à Brutus : évolution ou révolution?", Nouvelle Revue d'Onomastique, 1989, 13-14, pp.43-75)

6.Voir notamment Jacques BOUINEAU, Les toges du pouvoir ou la Révolution du droit antique (1789-1799), Toulouse, 1986, qui détaille en annexes le palmarès des différentes réminiscences antiques utilisées par les orateurs (pp.484-514).

7.Nous nous permettons de renvoyer à notre Doctorat pour un tour d'horizon détaillé des listes de référence et un index des noms relevés dans ces différentes sources. 8.La grande variabilité de motivation des choix de prénoms et l'importance d'une analyse au cas par cas ont été longuement développées dans la communication de Pierre-Henri Billy en 1997 au séminaire doctoral de l'Institut d'histoire de la Révolution française (reprise dans le présent volume). 
9.Par exemple Claude alias "Diagoras" alias "Demophile" Fauriel, agent national de la commune de Saint-Étienne, plus tard archiviste et historien, membre de l'Académie des Inscriptions et Belles-Lettres, qui déclare la naissance de Cornellie, fille de l'écrivain "Théophile" Tourron, le 17 thermidor an II.

10.Liberté figure déjà dans pas moins de quinze actes de baptême enregistrés à Marseille au cours de l'été 1790 (sans compter les enfants trouvés) et le choix de Brutus apparaît pour la première fois dès le 18 juillet de cette année là. Mais le phénomène s'est ensuite fortement raréfié pour ne reprendre qu'à l'extrême fin 1792. Sur les prénoms à Marseille pendant la Révolution, $c f$. Danielle MAURE, "Jean-Jacques Rousseau et Guillaume Tell nés à Marseille en 93 : Le hochet à l'épreuve", in Tout le portrait de son père. Des paroisses à l'état civil, Marseille, 1586-1889, Marseille, 1991, pp. 121-174.

11.Sur la situation parisienne : Raphaël BANGE, "Recherches sur les prénoms révolutionnaires à Paris", Annales historiques de la Révolution française, 1994, n¹, pp. 39-65.

12.Trois mois après la naissance de sa fille, prénommée Nièvre (Nevers, section du Levant, 11août 1793), le représentant du Peuple est encore sollicité pour rebaptiser un jeune garçon, à qui il attribue le prénom Brutus (même section, 1er brumaire an II). 13.Relevé effectué à partir de la bobine de microfilm 5 MI482/265, qui commence avec le registre de naissance ouvert le 19mars 1793. En ne retenant que les enfants dont les parents sont connus, ce dépouillement porte sur un total de 2065 actes (1062 filles, 1003 garçons).

14. Voir la contribution de Philippe Daumas dans ce même volume.

15.Alain CRoIX et Pascale ScIlBo éds., op cit., pp.29-33. Voir aussi Alain CRoIX, "Les prénoms révolutionnaires dans les campagnes de Loire-Inférieure”, Mélanges offerts à Jean Jacquart, Paris, 1994, p.156 et graphique p.162.

16.Pour valider cette méthode, il faudrait en outre prendre en compte une "marge d'erreur" de 10 à $20 \%$ puisque même des prénoms tout à fait traditionnels peuvent parfois être choisis sans rapport visible avec les noms de l'entourage (Marie 7 fois sur 57 à Cherbourg, Jacques 4 fois sur 25...). Ajoutons que dans un grand nombre de communes, l'absence de femmes parmi les témoins des déclarations de naissance empêche le recours à ce type d'analyse.

17.Seul registre parisien de cette époque à avoir échappé aux destructions, le Registre des baptêmes de la paroisse Saint-Roch pour 1790 est conservé aux Archives départementales de Paris sous la cote V6E/1. Tous rangs confondus, on y dénombre 31 attributions du nom Adélaïde sur un total de 284 actes de baptême (soit une forte proportion de 10,9\%, qui s'explique par la fréquence élevée des noms de baptême comportant deux ou trois unités). Parmi les autres prénoms féminins en progression dont la carrière est déjà bien entamée à cette date, notons aussi Joséphine (21 cas), Victoire (14), Alexandrine (10), Aimée et Augustine (8), Angélique, Julie, Sophie (7), etc. 18.Cette étude des prénoms des enfants parisiens se fonde sur un dépouillement exhaustif des actes de naissance reconstitués pour l'ensemble de cette période (A.D. Paris, série V2E, État civil reconstitué).

19.Jean-Pierre LETHUILLIER, "Prénoms et Révolution : enquête sur le corpus falaisien", Annales de Normandie, octobre 1989, 4, pp. 413-431; voir aussi sa communication sur les prénoms rennais figurant dans le présent volume.

20.Un acte de naissance d'Arras est en effet entaché d'une irrégularité manifeste : dans la déclaration de naissance de François Décadi Dauchet, enregistrée le 1er thermidor an II, l'officier d'état civil a "omis" de mentionner le nom de la personne lui ayant 
présenté l'enfant... En réalité, le père était alors sous les verrous : il s'agit de JeanBaptiste Dauchet, qui sera libéré après Thermidor et obtiendra ultérieurement un jugement de rectification en justice (du 5 fructidor anXII) dénonçant l'attribution de "François Décadi au lieu de François Benjamin qui avaient été désignés" à la sage-femme chargée de déclarer l'enfant. (Remarquons que le prénom de substitution n'aurait guère déparé le registre de naissance d'Arras, qui porte à la même date la déclaration d'autres prénoms équivoques comme Amaranthe, Narcisse et Virginie.) Or ce Dauchet d'Arras, jurisconsulte, est précisément le même homme que le député du Pas-de-Calais au Conseil des Cinq-Cent qui prend la parole à la tribune le 15 prairial an V, pour affirmer que bon nombre de pères de l'an II n'ont pas présenté leurs enfants aux officiers publics "parce que ceux-ci contraignaient à [leur] donner les noms odieux de Marat, de Robespierre ou d'autres chefs de la tyrannie..." (Moniteur, XXVIII, p. 723). L'accusation de Dauchet se fonde assurément sur son exemple personnel, légèrement travesti pour la circonstance (à l'époque du Directoire, Marat et Robespierre sont évidemment plus facile à diaboliser que le décadi), et généralisé de manière sans doute un peu artificielle... La discussion sera rapidement close par les députés, mais la dénonciation sera reprise et développée à l'envi par différents auteurs jusqu'au xxesiècle.

21.7 actes de l'état civil d'Arras portent en marge la mention d'un jugement de suppression ou remplacement d'un prénom révolutionnaire, 6 cas à Montpellier et autant à Nevers cela ne concerne guère plus de $1 \%$ des actes sur près de 1500 enfants ayant reçu un prénom révolutionnaire indiscutable dans l'une de ces trois villes entre 1793 et l'an III... Ces demandes de changement émanent presque toujours de familles de marchands, d'officiers ou de professions libérales, et nous renseignent bien davantage sur le contexte idéologique et social de l'après-Révolution que sur d'éventuelles contraintes exercées au moment du choix de ces prénoms.

22.Après la mise en place des officiers publics, le libellé de la première déclaration de naissance indique "les témoins ont donné à cet enfant pour prénoms...", mais sur les deux prénoms attribués, Félicité et Joséphine, le second est biffé sans explications. Le texte des actes suivants mentionne expressément le singulier ("ont donné pour prénom celui de...") et, de fait, tous les enfants déclarés à partir de cette date ne recevront plus qu'un unique prénom.

23.Le Titre III, Article 7 du décret instaurant l'état civil stipulait simplement que l'acte de naissance de l'enfant contiendra "le prénom qui lui sera donné", sans plus de précisions sur ce terme de prénom, pourtant inédit jusque-là dans le vocabulaire juridique français. Insistant sur le singulier, une brochure explicative parue à cette époque propose une interprétation restrictive du texte de loi : "Le Décret semble interdire la faculté de donner désormais à un enfant plusieurs prénoms, ce qui est assez sage, en ce que cela tend à éviter la confusion dans les généalogies" (État civil des citoyens, ou analyse sommaire du décret du 20 septembre..., Paris, 1793, p. 51.)

24.Sur plusieurs milliers d'actes enregistrés à Versailles entre 1793 et l'an IV, les seuls prénoms multiples acceptés à l'état civil, outre un Pierre Sansculottes de compromis, sont Marie Anne, Jean Baptiste, Jean Jacques, ainsi que des références révolutionnaires (Mutius Scevola, Guillaume Tell, Dix Août, Saint Fargeau) et certains noms de guerre (Belle Rose, Belle Fleur, Fleur d'Épine). S'y ajoute un cas isolé de François Nicolas dont le premier élément a été rajouté en marge, sans doute sur l'insistance du témoin, qui porte ce même double prénom. 
25.De son côté, tout en reconnaissant un caractère ambigu à Rose ou Marguerite, Serge Bianchi insistait sur la spontanéité des choix de prénoms à Corbeil, soulignée par un score global moyen de $48,7 \%$ de prénoms révolutionnaires ("Les prénoms “révolutionnaires" en l'an II l'exemple de Corbeil et de la Seine-et-Marne”, Le Prénom. Mode et histoire, Entretiens de Malher 1980, Paris, 1984, pp.255-270) ; alors qu'en ajoutant effectivement les Rose, Marguerite, Julienne, Auguste et autre Virginie il aurait obtenu un total voisin de $100 \%$ et aurait ainsi pu constater la disparition des prénoms traditionnels les plus courants. Gérard Cholvy donnait pour sa part dans l'excès inverse en présentant le cas de Montpellier : il souligne le caractère artificiel des choix de prénoms révolutionnaires, qui représentent selon lui 100\% des naissances en floréal an II ("Une Révolution culturelle Le test des prénoms", Pratiques religieuses, mentalités et spiritualités dans l'Europe révolutionnaire, Actes du Colloque international de Chantilly, Turnhout, 1988, pp.300-306), sans préciser que pour arriver à un tel total il faut considérer comme prénoms de rupture des choix modérés tels que Hector, Rose, Hyacinthe, Angélique..., dont il s'est pourtant attaché précédemment à démontrer le caractère traditionnel. En tout état de cause, les cas de Corbeil et Montpellier s'avèrent être des phénomènes généralisés de type 4 , cas exceptionnels qui ne peuvent guère prétendre être représentatifs de ce qui se passe dans le reste du pays...

26.Ces villes font partie du corpus de communes dont nous avons dépouillé l'état civil dans le cadre de nos recherches de doctorat, à l'exception d'Agen (où nous avons dû nous contenter de sondages partiels) et de Beaune, étudié dans Henri-Antoine PETIT, "Les prénoms révolutionnaires dans le district de Beaune", Recueil des travaux du Centre beaunois d'études historiques, 1992, 11, pp.63-74 (pp. 66-69 pour la ville de Beaune). 27.Si on en juge par les listes et résultats figurant dans Christian CAMPS, "Les prénoms révolutionnaires à Agde et Marseillan (Hérault)”, Nouvelle Revue d'Onomastique, 1989, 13-14, pp.131-154.

28.Pierre EXBRAYAT, "Prénoms républicains à Largentière", Communautés d'Oc et Révolution française, Actes du 58e Congrès de la Fédération des Sociétés historiques du Languedoc méditerranéen et du Roussillon, Aubenas, 1987, II, pp.187-193.

29.Jean-François PÉRol, "Le "fricotage" de "Verge d'or - caille-lait" avec "Belle de nuit sans culote" !", Lemouzi, 74, 1980, 125-140.

30.Jean DonAT, "Une application du calendrier républicain aux inscriptions à l'état civil", Mémoires de l'Académie des sciences, inscriptions et belles-lettres de Toulouse, 1933, XI, pp. 83-107.

31.D’après le Registre des délibérations du Comité de surveillance d'Aigueperse (A.D. Rhône, section ancienne, $33 \mathrm{~L}^{*}$ ). Pour une analyse du cas de Villefranche, nous renvoyons à notre communication "Révolution et mentalités : les prénoms à Villefranche-sur-Saône, capitale du Beaujolais", dans Ville et Révolution française, Bruno BENOIT éd., Actes du Colloque international de Lyon, mars 1993, Lyon, 1994, pp. 273-286.

32.DE RIBIER (Dr), “Onomastique révolutionnaire à St Bonnet-de-Salers et à Aurillac”, Revue de la Haute Auvergne, 1935-1936, XXXVII-XXXVIII, pp. 283-285.

33.Louis PEROUAS et al., Léonard, Marie, Jean et les autres, les prénoms en Limousin depuis un millénaire, Paris, 1984 (cf. p. 138).

34.Pierre ARCHES, “Les prénoms révolutionnaires à Gourgé”, Bulletin de la société historique et scientifique des Deux-Sèvres, 1983, XV, nº1, p. 63.

35.Michel LE PESANT, "Prénoms révolutionnaires et contre-révolutionnaires dans l'Eure, 1792-1795", Bulletin de la société des antiquaires de Normandie, 1969, LVIII, pp. 482-489 [p. 485]. 
36.Jean-Pierre PELISSIER, “Les Romarins ancêtres des Jules : les prénoms agricoles des ans II et III à Mézilles (Yonne)", Mesurer et Comprendre, Mélanges offerts à Jacques Dupâquier, Paris, 1993, pp.435-448.

37.Serge BIANCHI, "Enquête sur 8000 prénoms", 89 en Essonne, mars-avril 1990, n 6, pp. 62-70 Albert Vion, "Les prénoms républicains dans le Pas-de-Calais”, Bulletin de la Commission départementale d'histoire et d'archéologie du Pas-de-Calais, 1989, t. 12, 4, pp. 385-398. Voir aussi Albert Vion, "Les prénoms républicains dans le district de Calais", Bulletin historique et artistique du Calaisis, 116-117, déc. 1988 - mars 1989, pp.277-295, dont les tableaux permettent de déduire que les deux tiers des communes de ce district ont vu l'attribution d'au moins un prénom républicain entre 1793 et l'an III.

38.Jacques BERNET, "Les prénoms républicains sous la Révolution française l'exemple du district de Compiègne, 1793-1795”, dans Le Prénom, mode et histoire, pp. 247-253 (cf. p. 250) ; AlineTRABUT, Égalité, Bourrache, Aristide et les autres... Les prénoms révolutionnaires dans le département des Côtes-du-Nord (1789-1800), Saint-Brieuc, 1993, 263 p. (en particulier p. 82). Précisons que ces deux études s'appuient sur des sondages partiels (dépouillements limités à une soixantaine de communes) et que ces estimations mériteraient sans doute d'être revues légèrement à la baisse.

39.Henri-Antoine PeTit, “Les prénoms révolutionnaires en Côte d'Or”, Annales de Bourgogne, 1990, 62, pp.62-72, notamment p.71 : “Sur les 355 communes étudiées, 62 seulement comptent des prénoms révolutionnaires et, souvent, ceux-ci ne concernent qu'un ou deux enfants. (...) Si les 740 communes du département avaient été étudiées, les pourcentages seraient beaucoup plus faibles, les petites communes écartées ne comptant qu'un nombre infime de prénoms révolutionnaires."

\section{RÉSUMÉS}

Une analyse comparative menée en parallèle sur une cinquantaine de grandes villes françaises et sur l'ensemble des 133 communes d'un district rural (Villefranche-en-Beaujolais) conduit à dégager une typologie des attributions de prénoms au cours de la Révolution, et ainsi à mieux cerner le phénomène des "prénoms révolutionnaires" qui se manifeste aux quatre coins du pays, principalement entre 1793 et l'anIII. Illustré par une première série de graphiques, le suivi des seuls cas indiscutables, prénoms dénués d'équivoque comme par exemple Floréal ou Liberté, permet déjà de repérer de fortes variations d'une ville à l'autre dans la fréquence et la périodisation des choix de prénoms révolutionnaires. Mais l'élargissement de l'enquête à d'autres catégories de prénoms, répondant à une détermination plus mitigée (Victoire, Rose, Aimé...) ou à des modes apparemment dénuées de caractère idéologique (Caroline, Laure...), s'avère indispensable pour restituer le phénomène dans sa diversité et notamment pour mettre en évidence quelques cas extrêmes de mouvements collectifs généralisés, dans des communes où les prénoms exclusivement traditionnels comme Jean Joseph ou Marie Madeleine se trouvent systématiquement écartés des déclarations de naissance pendant plusieurs mois. Un tel résultat incite à remettre localement en question la spontanéité et la liberté de choix des parents. Étendue à l'ensemble des communes étudiées, une statistique globale donne la répartition des 
différents types de situation, témoignant de l'inégale réception des prénoms révolutionnaires par les Français de l'an II.

First names at a critic period of the french Revolution: a typological study of revolutionary and non-christian first names in France during AnII (1793-1794).

Comparative analysis of data, obtained from the study of registers of births from near fifty French towns and from 133 rural communities of the district of Villefranche-en-Beaujolais, led to define a typology of children's first names during the French Revolution, especially of the "prénoms révolutionnaires" given during the years an II-an III. As seen from the graphic representation, there was a good lot of peculiarities from a city to another, either as relative number or chronological periodization, when considering only the names truly and indisputably linked to the revolutionary circumstances (as Floréal or Liberté for example). Nevertheless, the study would stand uncompleted unless taking in due consideration other sets of names, although their ties with revolutionary ideology were less apparent (Victoire, Rose, Aimé...), or even absent (Caroline, Laure...). When doing so, the complexity of the first names problem was fully documented in a few cities, at least for some months, the lack of some Christian names among those most used before the Revolution (Jean Joseph, Marie Madeleine...) was a striking phenomenon, and one is compelled to cast doubt about parent's freedom in naming their children. Using the typology so defined, a recapitulative statistics established for the whole set of data demonstrated the unequal reception of revolutionary first names in the French population during the crucial years of the Revolution.

\section{INDEX}

Mots-clés : prénoms révolutionnaires, villes françaises, Beaujolais, phénomène collectif, typologie

\section{AUTEUR}

\section{RAPHAËL BANGE}

I.H.R.F. - Université de Paris I Panthéon-Sorbonne 